\title{
Methotrexate-Induced Toxic Epidermal Necrolysis: A Rare Case Report and Review of Literature
}

\author{
Pritam Kataria, Pradip Kendre, Apurva Patel ${ }^{1}$, Nahush Tahiliani, Sushant Ikhar ${ }^{1}$ \\ Departments of Medical and Paediatric Oncology and 'Medical Oncology, Gujarat Cancer and Research Institute, Ahmedabad, Gujarat, India
}

\section{Abstract}

Acute lymphoblastic leukemia (ALL) is the most common malignancy in pediatric patients, and it is characterized by the presence of malignant lymphoblasts within the bone marrow and peripheral blood. The treatment of ALL involves induction, consolidation, reinduction, and maintenance therapy. Consolidation therapy in ALL-Berlin-Frankfurt-Münster 90 protocol involves the use of high-dose methotrexate (HDMTX, $5 \mathrm{~g} / \mathrm{m}^{2}$ ) over $24 \mathrm{~h}$ as continuous infusion. The adverse effects due to HDMTX include renal dysfunction in $2 \%-12 \%$ patients, which can lead to increased systemic MTX exposure, leading to further myelosuppression, mucositis, hepatotoxicity, skin toxicity, and, in severe cases, multiorgan failure. Dermatologic toxicity due to MTX includes morbilliform drug rash, photoreactivation, photoenhancement, and skin hyperpigmentation. Stevens-Johnson syndrome and toxic epidermal necrolysis (TEN) are rare and possibly fatal reaction which can occur with MTX. Here, we describe a patient with B-cell ALL who developed TEN after administration of HDMTX.

Keywords: Acute lymphoblastic leukemia, methotrexate, toxic epidermal necrolysis

\section{INTRODUCTION}

Acute leukemia is the most common form of cancer in children which accounts for approximately $30 \%$ of all childhood malignancies. High-dose methotrexate (HDMTX, $5 \mathrm{~g} / \mathrm{m}^{2}$ ) forms the cornerstone of treatment of the consolidation therapy in Berlin-Frankfurt-Münster (BFM) 90 acute lymphoblastic leukemia (ALL) protocol which includes four doses at the interval of 14 days. Toxic epidermal necrolysis (TEN) is a life-threatening disease which is characterized by extensive destruction of the epidermis. The mortality rate ranges from $25 \%$ to $30 \%$ due to septicemia and various metabolic disturbances. The pathogenesis underlying TEN is an adverse drug reaction to specific toxic metabolites. ${ }^{[1]}$ We describe in this report a fatal case of HDMTX toxicity in case of ALL.

\section{Case Report}

A 20-year-old young adult male patient suffering from ALL presented to the emergency department with exfoliation of skin over the face, neck, trunk, limbs, oral cavity and decrease in urine output 1 day before the admission. The patient was apparently alright 1 day back when he developed the above-said symptoms. After detailed history and review

\begin{tabular}{|l|l|}
\hline \multicolumn{3}{c|}{ Access this article online } \\
\hline Quick Response Code: & Website: \\
& www.ijccm.org \\
& \\
\end{tabular}

of previous medical records, the patient was a known case of ALL on BFM 90 ALL protocol. As per the schedule, the patient had received HDMTX $\left(5 \mathrm{~g} / \mathrm{m}^{2}\right)$ just 5-6 days back. After the HDMTX, the patient had received only two doses of injectable leucovorin rescue. The patient took discharge against medical advice (DAMA), pending further leucovorin and methotrexate levels. At the time of DAMA, serum MTX levels were high. Subsequently, the patient was apparently alright for 3 days when he noticed discoloration of the skin followed by exfoliation over the face, neck, trunk, limbs, and oral cavity. Subsequently, the patient developed nonprojectile vomiting and decreased urine output. The patient was referred by the local physician to the medical oncologist for further management. At the time of admission, the patient was conscious, cooperative well oriented in time, place, and person. The vital parameters were as follows: blood pressure of $100 / 70 \mathrm{mmHg}$, pulse $100 / \mathrm{min}$, respiratory rate $16 / \mathrm{min}$, and abdomino-thoracic type. On systemic examination,

Address for correspondence: Dr. Apurva Patel, Department of Medical Oncology, Gujarat Cancer and Research Institute, Ahmedabad - 380 016, Gujarat, India. E-mail: shrutavpatel@rediffmail.com

This is an open access journal, and articles are distributed under the terms of the Creative Commons Attribution-NonCommercial-ShareAlike 4.0 License, which allows others to remix, tweak, and build upon the work non-commercially, as long as appropriate credit is given and the new creations are licensed under the identical terms.

For reprints contact: reprints@medknow.com

How to cite this article: Kataria P, Kendre P, Patel A, Tahiliani N, Ikhar S. Methotrexate-induced toxic epidermal necrolysis: A rare case report and review of literature. Indian J Crit Care Med 2018;22:740-2. 
the patient was febrile, there were signs of dehydration, and there were exfoliation and blebs over the skin (face, neck, trunk, limbs, and perianal area) and in within the oral cavity covering $>30 \%$ body surface area [Figure 1]. Blood investigation suggested pancytopenia (hemoglobin $-7 \mathrm{~g} / \mathrm{dl}$, total leukocyte count-1000/UL, platelet count-35,000/UL), renal failure (serum creatinine and blood urea nitrogen of 8 and $90 \mathrm{mg} / \mathrm{dl}$, respectively), and elevated serum MTX levels (2 U/L at the end of $144 \mathrm{~h}$ ). Blood culture was sent and the patient was started on supportive antibiotics, i.e., injection cefoperazone + injection sulbactam $3 \mathrm{~g}$ intravenous (iv) twice a day, injection linezolid $600 \mathrm{mg}$ iv twice a day, and injection clindamycin $600 \mathrm{mg}$ iv thrice a day. In view of decreased counts, injection filgrastim $300 \mu \mathrm{g}$ subcutaneously once a day was given. Hydration was maintained at the rate of $75 \mathrm{mg} / \mathrm{m}^{2}$ with addition of $40 \mathrm{mEq} / \mathrm{L}$ sodium bicarbonate to alkalinize the urine (maintain urine $\mathrm{pH}$ of 7 or greater) as per the JVP measured using the central line. Injection leucovorin $15 \mathrm{mg}$ iv 6 hourly was started. Dialysis support was given in view of deranged creatinine. However, the patient became unconscious the next day and further developed progressively increasing renal failure with creatinine $10 \mathrm{mg} / \mathrm{dl}$ and decrease counts (absolute neutrophil count - 800) and electrolyte disturbance. The patient was further started on injection caspofungin $70 \mathrm{mg}$ as loading dose followed by $50 \mathrm{mg}$ in subsequent dose. The patient was further continued dialysis on the next day. However, the patient progressively developed liver dysfunction with deranged bilirubin with total bilirubin of $4.8 \mathrm{mg} / \mathrm{dl}$ and serum glutamic oxaloacetic transaminase/serum glutamic pyruvic transaminase of 332/432, with deranged electrolytes and deranged serum creatinine of $10.5 \mathrm{~g} / \mathrm{dl}$, and succumbed on the $3^{\text {rd }}$ day in spite of all best measures being taken.

\section{Discussion}

MTX forms an important part of treatment regimen of various malignancies. HDMTX is used to treat a range of adult and childhood cancers notably ALL, primary central

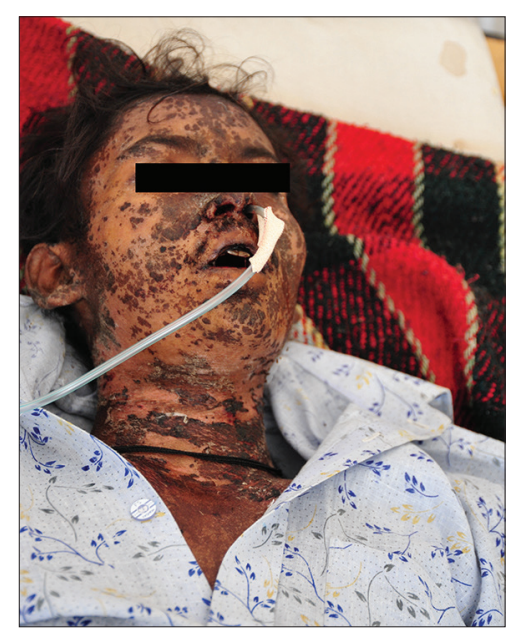

Figure 1: Image showing extensive skin desquamation nervous system lymphoma, mantle cell lymphoma (as part of HyperCVAD regimen), and osteosarcoma. ${ }^{[2-4]}$

MTX is dihydrofolate reductase inhibitor whereby it interferes with DNA synthesis leading to cell death. ${ }^{[5-8]}$

Although HDMTX is safely administered to most patients, it can lead to different adverse events such as nephrotoxicity, hepatotoxicity, gastrointestinal side effects, mucositis, and dermatologic toxicity.

Increased levels of MTX have been seen with folic acid deficiency or by medications such as barbiturates, nitrofurantoin (impairs folic acid absorption), trimethoprim-sulfamethoxazole, triamterene, pyrimethamine and phenytoin, probenecid, salicylates, and sulfonamides (competes with MTX for laumin binding and displaces MTX). ${ }^{[9]}$

Dermatologic adverse events such as mucositis, urticaria, angioedema, photosensitivity, alopecia, maculopapular eruption, erythema, desquamation, Stevens-Johnson syndrome, TEN, and erosion of psoriatic plaques have been reported as adverse cutaneous reactions to MTX.

It is still debatable whether the epidermal necrolysis is an allergic or dose-related toxicity reaction. Various dermatologic manifestation has been described in literature by Copur et al. (generalized maculopapular eruption), ${ }^{[10]}$ Lawrence and Dahl (ulceration on psoriatic plaques and pre-existing dermatitis), ${ }^{[1]}$ and Martins da Cunha et al. (palmoplantar erythema and desquamation) ${ }^{[12]}$ MTX-induced TEN has been described in literature either as hypersensitivity reaction, direct cell toxicity, or interaction with drug such as nonsteroidal anti-inflammatory drugs (NSAIDs).

Treatment of TEN involves stopping the drug, supportive care, fluid and electrolyte balance, feeding, topical antiseptics, and dressing of denuded skin, antacids, analgesics, anxiolytics, and antipyretics.

To avoid MTX-related adverse events, various steps have been advised such as having normal renal and hepatic function before the start of therapy, adequate hydration and timely start of leucovorin rescue, monitoring of serum MTX levels as a prerequisite of discharge and avoiding drugs such as cephalosporins, NSAIDS, proton pump inhibitors, trimethoprim-sulfamethoxazole, sulfonamides, and salicylate, and reduction of MTX dosage as and when required.

\section{Conclusion}

MTX though known to exhibit different skin manifestation has been rarely reported in literature. It is a life-threatening situation and supportive therapy with dialysis in case of renal failure forms the mainstay of therapy. This case has been presented to highlight this known but rarely reported adverse side effect of the drug so that precautionary measures can be taken to avoid it though not completely preventable since pathogenesis of various dermatologic manifestations is manifold. 


\section{Financial support and sponsorship}

Nil.

\section{Conflicts of interest}

There are no conflicts of interest.

\section{RefEREnCeS}

1. Fritsch PO, Sidoroff A. Drug-induced Stevens-Johnson syndrome/toxic epidermal necrolysis. Am J Clin Dermatol 2000;1:349-60.

2. Stoller RG, Hande KR, Jacobs SA, Rosenberg SA, Chabner BA. Use of plasma pharmacokinetics to predict and prevent methotrexate toxicity. N Engl J Med 1977;297:630-4.

3. Mitchell MS, Wawro NW, DeConti RC, Kaplan SR, Papac R, Bertino JR, et al. Effectiveness of high-dose infusions of methotrexate followed by leucovorin in carcinoma of the head and neck. Cancer Res 1968:28:1088-94.

4. Abrey LE, DeAngelis LM, Yahalom J. Long-term survival in primary CNS lymphoma. J Clin Oncol 1998;16:859-63.

5. Ackland SP, Schilsky RL. High-dose methotrexate: A critical reappraisal.
J Clin Oncol 1987;5:2017-31.

6. Goldman ID, Matherly LH. The cellular pharmacology of methotrexate. Pharmacol Ther 1985;28:77-102.

7. Green MR, Chowdhary S, Lombardi KM, Chalmers LM, Chamberlain M. Clinical utility and pharmacology of high-dose methotrexate in the treatment of primary CNS lymphoma. Expert Rev Neurother 2006;6:635-52.

8. Meyers PA, Flombaum C. High-dose methotrexate-induced renal dysfunction: Is glucarpidase necessary for rescue? J Clin Oncol 2011;29:e180.

9. Olsen EA. The pharmacology of methotrexate. J Am Acad Dermatol 1991;25:306-18.

10. Copur S, Dahut W, Chu E, Allegra CJ. Bone marrow aplasia and severe skin rash after a single low dose of methotrexate. Anticancer Drugs $1995 ; 6: 154-7$

11. Lawrence CM, Dahl MG. Two patterns of skin ulceration induced by methotrexate in patients with psoriasis. J Am Acad Dermatol 1984;11:1059-65.

12. Martins da Cunha AC, Rappersberger K, Gadner H. Toxic skin reaction restricted to palms and soles after high-dose methotrexate. Pediatr Hematol Oncol 1991;8:277-80. 